\title{
Experimental Investigation of a Rising Bubble in Aqueous Gelatin Solution under Gelation Process
}

\author{
Shuichi IwatA ${ }^{*} \uparrow$, Yasuhiro SAIKI", Ryo NAGUMo", Hideki MorI",

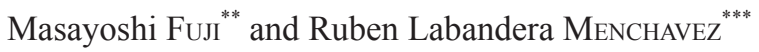 \\ "Graduate School Materials Science and of Engineering, Nagoya Institute of Technology, \\ Gokiso, Showa, Nagoya, 466-8555, Japan \\ *** Advanced Ceramics Research Center, Nagoya Institute of Technology, \\ 3-101-1, Honmachi, Tajimi, Gifu, 507-0033, Japan \\ ${ }^{* * *}$ Ceramic, Metallurgical and Mining Department, College of Engineering, MSU-Iligan Institute of Technology, \\ Tibanga, Iligan City 9200, Philippines \\ (Received : November 17, 2017)
}

\begin{abstract}
We have observed a progression of the shape and velocity of a bubble rising in aqueous gelatin solution under gelation process. Two experimental systems were designed to produce two different bubble sizes that were allowed to rise in test sections filled with aqueous gelatin solution. The physical gelling was allowed to proceed slowly in a temperature-controlled cooling system. The viscoelasticity of the aqueous gelatin solution was measured using a stress controlled rheometer under the same temperature history with the bubble rising observations. It was found that the change in bubble shape corresponds to the elastic behavior of solution due to the progress in gelation process. The gel point of the gelatin solution was conveniently determined by detecting the change in the shape of the rising bubble from a sphere shape to prolate and cuspidal shape. It was further observed that a small bubble is preferable during measurement because of its less impact on the gelation structure around the bubble. Subsequently, the measuring system designed in the present study is simple and convenient to install for offline process monitoring.
\end{abstract}

Key Words: Bubble motion / Cuspidal shape / Aqueous gelatin solution / Viscoelastic fluid

\section{INTRODUCTION}

The gelation process of polymer-based solutions whether natural or synthetic form is often involved with the transformation of a thermally heated mixture solution of macromolecules or particles into a solid-like material by the cooling process. The use of the solution-based polymer mixture results in various products using gelation process for applications in food, medicine, cosmetics, and others. The gelatin is widely used in various industries among others ${ }^{1)}$, which is a biopolymer derived from denatured collagen. For example, the gelatin is commonly applied in pharmaceutical products to contain drugs in hard or soft capsules. Further applications are also observed in adhesive for laminating materials, cosmetics and foods, such as jelly.

Among the aforementioned applications of gelatin, the coating and containment of drugs with non-toxic and thermoreversible material gains strong interest in medicine. This

\footnotetext{
$\dagger$ Corresponding author.

E-mail : iwa@nitech.ac.jp

Tel: +81-52-735-5256; Fax: +81-52-735-5255;
}

containment of drugs appear in a form of a soft capsule that is produced by the following procedure. At first drug as the inner fluid and gelatin as the outer fluid are passed through the cooling solution from a double nozzle. In the next step, the solutions flowed out from the double nozzle break up into a compound droplet. Finally, the capsule is formed by cooling and gelling of the outer gelatin solution of the droplet.

The success of producing the soft capsules relies on the optimum characteristics of the droplet. Several numerical analyses of this compound droplet formation have been reported $^{2,3)}$. To produce the compound drops, the capillary waves on the inner and outer interfaces of the solutions need to be coordinated. The capillary waves are identified in a narrow window of flow-rate and viscosity ratios in which it can be achieved. Thus, the viscosity ratio was found to be one of the important parameters to achieve the desirable compound drops. This finding was conducted in an isothermal system, in other words, assuming no change in physical properties of inner-middle-outer fluids. In practice, however, the gelation of gelatin solution proceeds gradually through a viscous change during cooling. Therefore, to determine the desirable 
operating conditions for the production of compound drops at acceptable quality, an in-situ measurement of viscoelasticity and gelation behavior of the solution during cooling is essential.

Generally, a rheometer is employed to measure the viscoelasticity and gelation behavior of polymer solutions. However, the rheometric method can potentially stress the developing macromolecules of gelatin during testing which may alter the information about its actual behavior during in-situ solidification ${ }^{4)}$.

The problem of the formation of the compound drop in gelatin solution is similar to the bubble dynamics in viscoelastic fluids. The shape of the rising bubbles is strongly affected by the type of fluids. In Newtonian fluid, the shape is spherical whereas in non-Newtonian or viscoelastic fluid the shape of the bubble is significantly deformed ${ }^{5)}$. This area of research is extensively studied and reviewed in the literature ${ }^{6,7)}$, which presents moderate disturbance in the solution matrix.

The present study is involved with monitoring the progression of the shape and velocity of a rising bubble in non-Newtonian fluid using a gelatin solution under gelation process. The experiment was conducted on a pre-designed system subjected to operating parameters such as bubble size, cooling temperature, flow rate, and others. These parameters were empirically determined and expected to have strong influence on the viscoelastic properties of the fluid and the bubble motion. We have designed the system that carried out in-situ measurement of gelation by monitoring the bubble behavior. The designed system is inexpensive and conveniently introduced to the production line. Lastly, the operating procedure of the designed system is relatively easy as compared to the conventional rheometric method.

\section{EXPERIMENTS}

\subsection{Preparation of gelatin aqueous solution}

In this study, we have used aqueous gelatin solution that is a transparent liquid to allow clear observation of a rising bubble. The gelatin is a protein prepared from partial hydrolytic degradation of a collagen ${ }^{8}$. A gelatin solution has the characteristic of easy physical gelation by cooling. Its gelation behavior is a transition from a type of random coil to a helix. The first step is the formation of the nucleation of a triple helix. The second step is the growth of this triple helix followed by entanglement between their helixes. Experiments were carried out using $3.0 \mathrm{wt} \%$ gelatin in aqueous solution. The aqueous solution was prepared from a gelatin powder (Wako Pure Chemical Ind., Lot No. LAL2187, WEF3618), which was mixed and dissolved in distilled water in the hot bath $\left(60^{\circ} \mathrm{C}\right)$ for an hour. Then, the mixed solution was cooled down in the water bath to $33-34{ }^{\circ} \mathrm{C}$ and further mixed for additional half an hour.

\subsection{Equipment design and bubble-rise experiment}

The experimental equipment mainly consisted of a cell for observation with a single bubble injection system, a thermostatic chamber and a high-resolution video camera. The injection system was capable of generating air bubbles with volumes between $5 \mathrm{~mm}^{3}$ and $800 \mathrm{~mm}^{3}$. Two experimental equipment were assembled as described below.

Figure 1 shows the prototype of the experimental equipment producing air bubbles with volumes between $5 \mathrm{~mm}^{3}$ and $40 \mathrm{~mm}^{3}$. The dimension of the cell for observation is as follows: $H=150 \mathrm{~mm}$ (Height) $\times W=80 \mathrm{~mm}$ (Width) $\times D$ $=37 \mathrm{~mm}$ (Depth). This cell was made of polystyrene under which the bubble injection device was installed. This device can efficiently control the injection of a single bubble into the aqueous solution of gelatin by pressure fluctuation. The fluctuation was effected in upside set-up of the cell on a speaker cone that emanated a single vibration ${ }^{9)}$.

Figure 2 exhibits the prototype of the equipment that produced volumes of air bubbles between $100 \mathrm{~mm}^{3}$ and $800 \mathrm{~mm}^{3}$. The cell was rectangular with dimensions of $H=$ $200 \mathrm{~mm}$ (Height), $W=100 \mathrm{~mm}$ (Width), and $D=50 \mathrm{~mm}$ (Depth). The bubble injection system was used to store a predetermined amount of air in a hemispherical cup within the cell. It was then possible to inject a single bubble into the solution by rotating the hemispherical cup. The thermostatic chamber was filled with distilled water. The temperature of the distilled water in the thermostatic chamber was adjusted by a cooling system. The temperature of the aqueous gelatin solution in the cell was also controlled to be the same as the temperature in the rheometer. We have filmed the generated shadow of the bubble with a high-resolution camera (60 fps in Tele Macro) by irradiating a strobe light at about $20 \mathrm{~Hz}$.

\subsection{Measurement of a dynamic viscoelasticity of the aqueous solution of gelatin by a rheometer}

To grasp the viscoelasticity and gelation behavior of the aqueous gelatin solution, we have performed a comparative evaluation using a shear oscillation on a rheometer (RS600, Thermo corp.). The rheometer was equipped with a cone plate sensor having a diameter of $60 \mathrm{~mm}$ and a cone angle of 2 degrees. The set-up was protected with a glass enclosure to prevent the sample edge from drying out during measurement. The measurement conditions were as follows: given strain, $\gamma=1.0[-]$, angular rate, and $\omega=1.0 \mathrm{rad} / \mathrm{s}$. The 


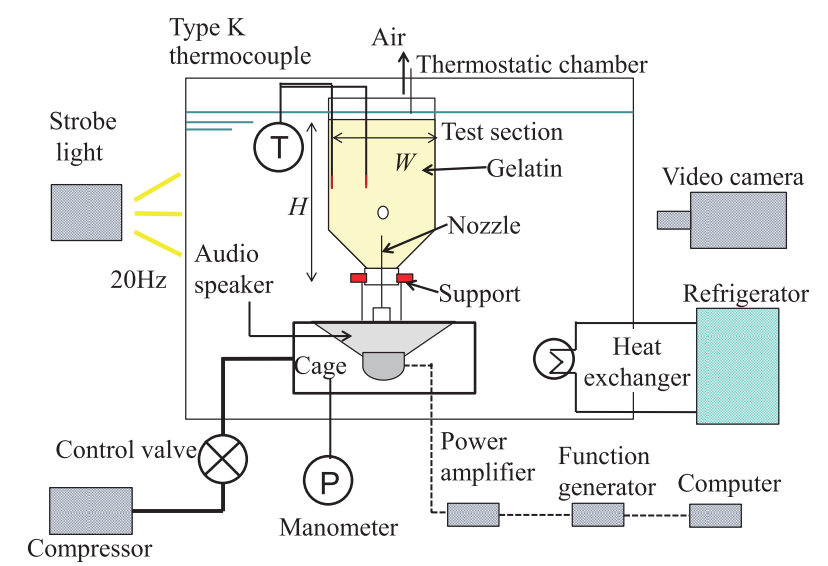

Fig. 1 Experimental apparatus for generating a small bubble $\left(V<40 \mathrm{~mm}^{3}\right)$.

Table I Experimental data of bubble $\left(5 \mathrm{~mm}^{3}\right)$ cooled at $26^{\circ} \mathrm{C}$ (Run 1$)$.

\begin{tabular}{|c|c|c|c|c|}
\hline No. & $\begin{array}{c}\text { Time } \\
t[\mathrm{~s}]\end{array}$ & $\begin{array}{c}\text { Velocity } \\
u[\mathrm{~mm} / \mathrm{s}]\end{array}$ & $\begin{array}{c}\text { Volume } \\
V\left[\mathrm{~mm}^{3}\right]\end{array}$ & Bubble shape \\
\hline 1 & 3595 & 164 & 4.87 & Spherical \\
\hline 2 & 4800 & 160 & 5.01 & Spherical \\
\hline 3 & 5471 & 154 & 5.02 & Spherical \\
\hline 4 & 5894 & 150 & 4.98 & Spherical \\
\hline
\end{tabular}

temperature was controlled by two different procedures. The first procedure (A) was cooling down of the prepared mixture to $26^{\circ} \mathrm{C}$ at a cooling rate of $0.267^{\circ} \mathrm{C} / \mathrm{min}$ for 30 minutes. The sample was then kept at $26^{\circ} \mathrm{C}$. The second procedure (B) was cooling down of the prepared mixture to $23{ }^{\circ} \mathrm{C}$ at a cooling rate of $0.263{ }^{\circ} \mathrm{C} / \mathrm{min}$ for 38 minutes, and then the sample was kept at $23{ }^{\circ} \mathrm{C}$.

\subsection{Measurement of the gel point by the rheometer}

The gel point of the aqueous solution of gelatin was evaluated in terms of the viscoelastic properties of the gelling solution. Takahashi ${ }^{10,11)}$ has suggested a method of gel point determination involving loss tangent curves at different frequencies. These curves are expected to cross at a common gel point, $t_{c}$, representing the transition point of the viscous liquid at which the energy dissipation is high during gelation to an elastic solid with a low energy dissipation. Moreover, at the transition point, the loss tangent of the liquid becomes frequency independent that roughly indicates the gelation time $^{12)}$.

\section{RESULTS AND DISCUSSION}

\subsection{Observation of rising bubble in the aqueous solution of gelatin}

In this experiment, we have measured the diameter,

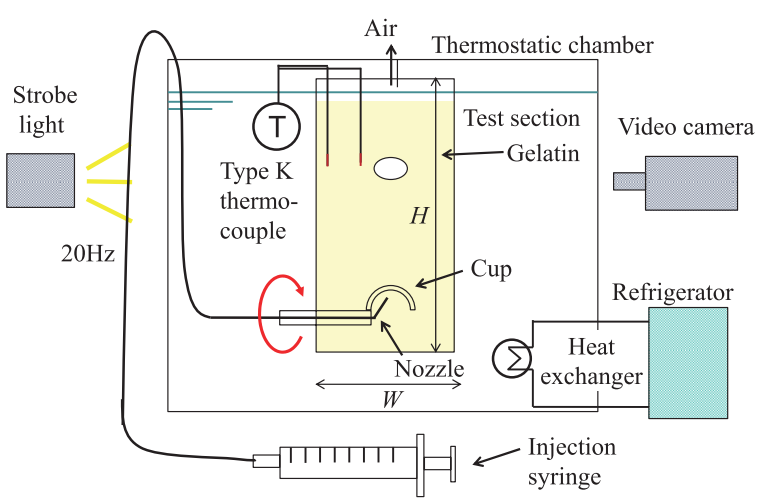

Fig. 2 Experimental apparatus for generating a bubble $\left(800 \mathrm{~mm}^{3}>V\right)$.

Table II Experimental data of bubble $\left(12 \sim 25 \mathrm{~mm}^{3}\right)$ cooled at $23{ }^{\circ} \mathrm{C}$ (Run 2).

\begin{tabular}{|c|c|c|c|c|}
\hline No. & $\begin{array}{c}\text { Time } \\
t[\mathrm{~s}]\end{array}$ & $\begin{array}{c}\text { Velocity } \\
u[\mathrm{~mm} / \mathrm{s}]\end{array}$ & $\begin{array}{c}\text { Volume } \\
V\left[\mathrm{~mm}^{3}\right]\end{array}$ & Bubble shape \\
\hline 1 & 3480 & 140 & 12.4 & Spherical \\
\hline 2 & 4200 & 90 & 15.2 & Spherical \\
\hline 3 & 5060 & 30.0 & 17.9 & Spherical \\
\hline 4 & 5340 & 3.80 & 20.9 & Prolate, Cusp \\
\hline 5 & 5840 & 2.70 & 24.4 & Prolate, Cusp \\
\hline
\end{tabular}

terminal velocity and shape of bubble when it reached at a position of about 5 or $10 \mathrm{~cm}$ above the nozzle. The horizontal and vertical diameters of the bubble, $d h$ and $d v$ respectively, were measured with a ruler installed parallel to the side walls of the cell. The rising velocity, $u$, was calculated from the moving distance measured at the travel time of 0.2 seconds or 10 seconds. Simultaneously, the entire process was filmed to monitor the shape of the bubble.

Table I lists the velocity and volume of the small bubble $\left(5 \mathrm{~mm}^{3}\right)$ cooled at $26^{\circ} \mathrm{C}$ as a function of the time elapsed. This experiment was denoted as Run 1. It was observed that the shape of the bubble remained spherical during the course of experiment as shown in Fig. 3. This is supported with a slightly decreasing velocity and volume of the bubble remained constant. These findings suggest that there has been no significant change in the physical properties of the solution undergoing the gelation process. This observation is consistent with the rheometric analysis (Section 3.2).

The resulting data using the same small bubble (12 24 $\mathrm{mm}^{3}$ ) flowed in the solution matrix (3.0 wt\% gelatin) but cooled at $23{ }^{\circ} \mathrm{C}$ were presented in Table II. This experiment was denoted as Run 2. It can be observed that the velocity is significantly decreasing while the bubble size is increasing and deforming with time. The deformation of the bubble 


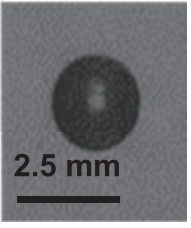

$t=3595 \mathrm{~s}$

$V=4.87 \mathrm{~mm}^{3}$

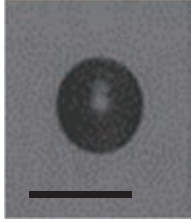

$t=4800 \mathrm{~s}$

$V=5.02 \mathrm{~mm}^{3}$

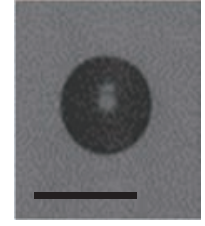

$t=5471 \mathrm{~s}$

$V=5.01 \mathrm{~mm}^{3}$

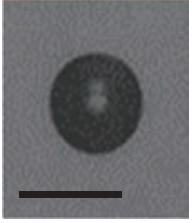

$t=5894 \mathrm{~s}$

$V=4.98 \mathrm{~mm}^{3}$

Fig. 3 Photographs of a rising bubble in $3.0 \mathrm{wt} \%$ aqueous gelatin solution at $26^{\circ} \mathrm{C}$ in Run 1 .

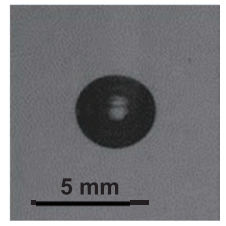

$t=3480 \mathrm{~s}$ $V=12.4 \mathrm{~mm}^{3}$

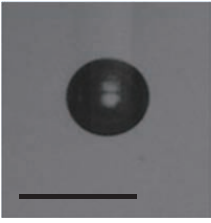

$t=4200 \mathrm{~s}$ $V=15.2 \mathrm{~mm}^{3}$

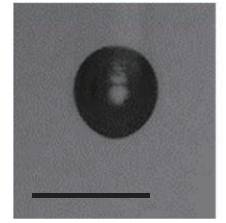

$t=5060 \mathrm{~s}$ $V=17.9 \mathrm{~mm}^{3}$

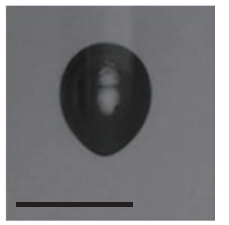

$t=5340 \mathrm{~s}$ $V=20.9 \mathrm{~mm}^{3}$

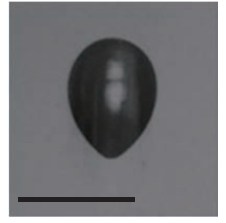

$t=5840 \mathrm{~s}$ $V=24.4 \mathrm{~mm}^{3}$
Fig. 4(a) Photographs of a rising bubble in $3.0 \mathrm{wt} \%$ aqueous gelatin solution at $23{ }^{\circ} \mathrm{C}$ in Run 2.

was visibly observed at about 5000 seconds as shown in Fig. 4(a). The growth of the bubble volume causes the transition of the bubble shape from spherical to spherical with a cusp at about 5300 seconds. Such deformation of the bubble was observed with a good repeatability. This suggests that at this event the elasticity of the gelling solution became dominant. At this stage, the viscoelasticity of the aqueous gelatin solution is contributed by the growth of the network structure formed by the gelation process. This process developed a normal stress that might act around the side and bottom of the bubble, leading to the shape of the bubble in prolate with the cusp (Fig. 4(a)). Ratio of the curvature radius at the top of the bubble, $R_{U}$, divided by the curvature radius at the bottom of the bubble, $R_{L}$, are plotted in Fig. 4(b). It can be observed that the ratio of curvature radius is significantly increasing after 5000 seconds. Taking into account the Laplace pressure and assuming uniform surface tension, such differences of the curvature radius corresponds to local liquid pressure difference between the top and the bottom of the bubble because gas pressure is uniform inside the bubble. The liquid pressure difference may indicate the viscoelasticity of the surrounding

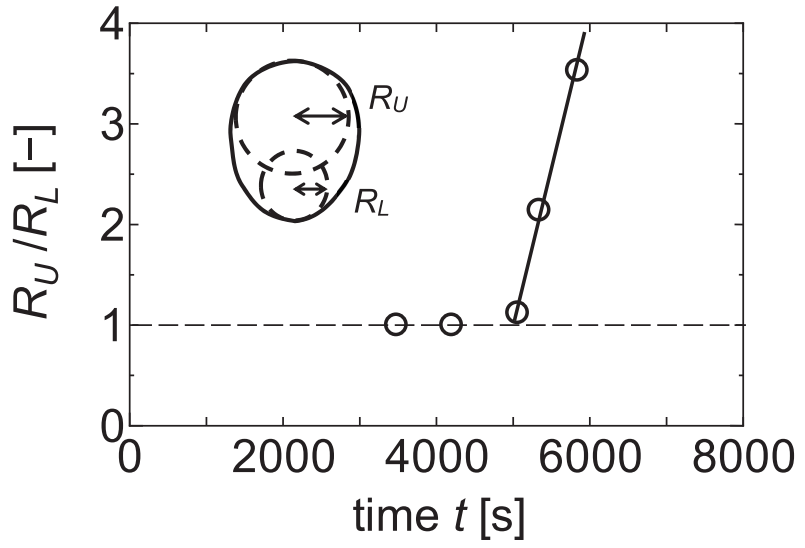

Fig. 4(b) Ratio of the curvature radius at the bottom of the bubble $R_{L}$ divided by the curvature radius at the top to the bubble $R_{U}$ in Run 2 .

fluid. Therefore, the changing point may corresponds to the beginning development of viscoelasticity.

Using the same condition $\left(T=23^{\circ} \mathrm{C}\right.$ and $3.0 \mathrm{wt} \%$ solution) but the bubble size is increased to $100 \mathrm{~mm}^{3}$, the experimental results are shown in Table III. This experiment was denoted as Run 3. It can be observed that the velocity is decreasing due to increasing viscosity of the solution at early stage of gelation. This corresponds to an oblate shape of the bubble with a relatively increasing volume as presented in Fig. 5. At about 4800 seconds of gelation, the bubble shape transformed to oblate shape with a long tail. Ohta et al. ${ }^{13)}$ mentioned that the long tail is caused by effect of elasticity of the solution. At larger volume, the bubble shape shifted to prolate with cusp and then restored to spherical shape with cusp (Fig. 5). This progression of the bubble shape was accompanied by the reduction of the velocity of the bubble due to high viscoelastic properties of the solution.

The observation of bubble dynamics was further conducted with a bubble volume of $800 \mathrm{~mm}^{3}$ at the same conditions ( $T=23{ }^{\circ} \mathrm{C}$ and $3.0 \mathrm{wt} \%$ solution). This experiment was denoted as Run 4 and the experimental results are presented 
Table III Experimental data of bubble $\left(100 \mathrm{~mm}^{3}\right)$ cooled at $23^{\circ} \mathrm{C}$ (Run 3).

\begin{tabular}{|c|c|c|c|c|}
\hline No. & $\begin{array}{c}\text { Time } \\
t[\mathrm{~s}]\end{array}$ & $\begin{array}{c}\text { Velocity } \\
u[\mathrm{~mm} / \mathrm{s}]\end{array}$ & $\begin{array}{c}\text { Volume } \\
V\left[\mathrm{~mm}^{3}\right]\end{array}$ & Bubble shape \\
\hline 1 & 3625 & 210 & 106 & Oblate \\
\hline 2 & 4460 & 200 & 93.4 & Oblate, Cusp \\
\hline 3 & 4800 & 215 & 165 & Oblate, Cusp \\
\hline 4 & 5400 & 170 & 117 & Prolate, Cusp \\
\hline 5 & 5860 & 155 & 120 & Spherical, Cusp \\
\hline
\end{tabular}

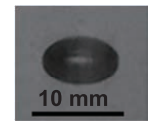

$t=3625 \mathrm{~s}$ $V=106 \mathrm{~mm}^{3}$

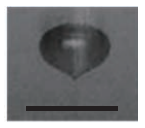

$t=4460 \mathrm{~s}$ $V=93.4 \mathrm{~mm}^{3}$
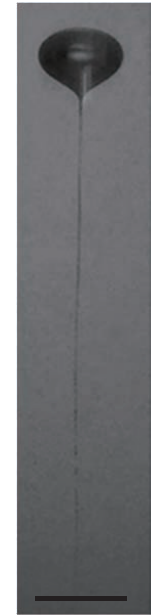

$t=4800 \mathrm{~s}$

$V=165 \mathrm{~mm}^{3}$

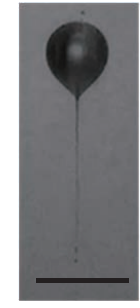

$t=5400 \mathrm{~s}$ $V=117 \mathrm{~mm}^{3}$ $V=120 \mathrm{~mm}^{3}$
Fig. 5 Photographs of a rising bubble in $3.0 \mathrm{wt} \%$ aqueous gelatin solution at $23{ }^{\circ} \mathrm{C}$ in Run 3.

in Table IV. It can be observed that the velocity of the bubble is not clearly changing with only slightly decreasing in volume at the early stage of gelation. At this stage of gelation, the viscosity was slightly rising, and the bubble was oblate in shape moving in wobbling motion as shown in Fig. 6. It can be hypothesized that at this event the inertial force was dominant. After 4200 seconds of gelation, the elasticity of the solution began to grow, causing the bubble to form an oblate shape with a cusp developed in a long tail.

Based on the experimental data above, we have found out that the change in the viscoelastic properties of the aqueous gelatin solution undergoing gelation process can be conveniently observed through the change in bubble motion. Moreover, it presents a simple and inexpensive method to clearly follow the gelation behavior of gelatin in solution.

\subsection{Comparative evaluation with the rheometric data}

Figures 7(a) and (b) show the results of shear oscillations measured on $3.0 \mathrm{wt} \%$ aqueous gelatin solution subjected to procedures A and B. As shown in Fig. 7(a), there is almost no observable change for both storage modulus, $G^{\prime}$,
Table IV Experimental data of bubble $\left(800 \mathrm{~mm}^{3}\right)$ cooled at $23^{\circ} \mathrm{C}$ (Run 4).

\begin{tabular}{|c|c|c|c|l|}
\hline No. & $\begin{array}{c}\text { Time } \\
t[\mathrm{~s}]\end{array}$ & $\begin{array}{c}\text { Velocity } \\
u[\mathrm{~mm} / \mathrm{s}]\end{array}$ & $\begin{array}{c}\text { Volume } \\
V\left[\mathrm{~mm}^{3}\right]\end{array}$ & Bubble shape \\
\hline 1 & 3557 & 225 & 754 & Oblate, Wobbling \\
\hline 2 & 4260 & 230 & 779 & Oblate, Cusp \\
\hline 3 & 5020 & 235 & 806 & Oblate, Cusp \\
\hline 4 & 5410 & 235 & 834 & Oblate, Cusp \\
\hline 5 & 5820 & 235 & 837 & Oblate, Cusp \\
\hline
\end{tabular}

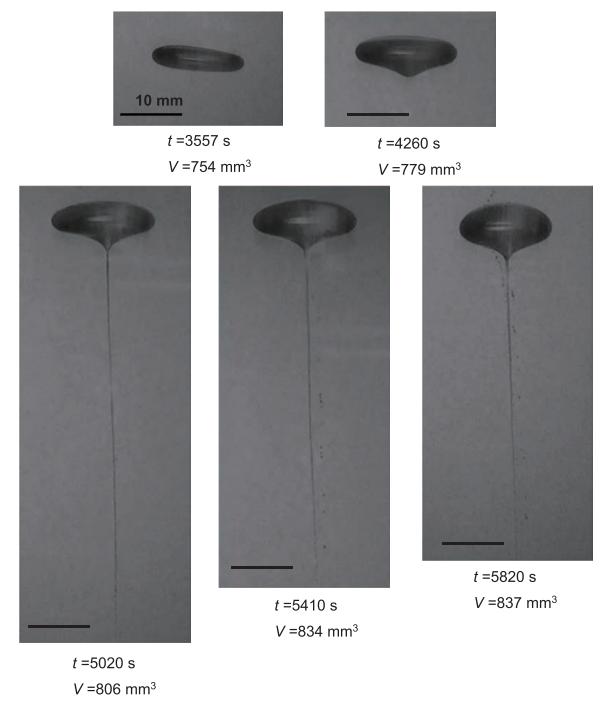

Fig. 6 Photographs of a rising bubble in $3.0 \mathrm{wt} \%$ aqueous gelatin solution at $23{ }^{\circ} \mathrm{C}$ in Run 4.

which represents the elastic response, and the loss modulus, $G^{\prime \prime}$, which represents the viscosity response. This finding indicates that the gelation did not occur in procedure A. On the other hand, Fig. 7(b) shows that the loss modulus, $G^{\prime \prime}$, rose gradually while the storage modulus, $G^{\prime}$, exhibited a constant value in the initial stage and then started to rise at a certain moment. This suggests that, in the early stage of gelation, the viscosity of the solution is increasing gradually while the elastic property is constant. This coincides with the formation of the triple helix structure that progresses gradually and extends the polymer chain. At this instance, the polymer chains are not yet entangled. In about 4200s after this event, the response of the elastic property began to be visible, since the entanglement started to grow. It can then be deduced that the gelation process occurred using the procedure B. Lastly, it was further observed that this measurement was reproducible.

Moreover, the knowledge of the gel point of the aqueous gelatin solution is important for comparative purposes. Hence, the experimental curves in Fig. 8 depicted the similar loss tangent, $\tan \delta$, profile versus gelling time for a solution subjected to different frequencies. It is clearly observed on 
the figure that all curves revealed a gelation time, $t_{c}$, of 5300 seconds as their common point of intersection. Lastly, the experimental data also provide information on the structural progression of the aqueous gelatin solution during the gelation process from the loss tangent curves.

It is also informative to superimpose the time change of the shape of the bubbles on the loss tangent curves as shown in Fig. 8. It can be observed on the figure that the shape of the rising small bubbles $\left(12 \sim 24 \mathrm{~mm}^{3}\right)$ changes from spherical or oblate to the cusp shape at the gelation point. The shape of rising large bubbles $\left(100 \sim 800 \mathrm{~mm}^{3}\right)$ shift from oblate to the cusp shape at the point where the tangent delta starts to fall due to the start of the growth of the elastic property.

Moreover, it was found out that there is a possibility to confirm any change of the solution properties about elasticity by observing a change in the bubble shape. For a small bubble volume (12 24 $\left.\mathrm{mm}^{3}\right)$, the time (5000 seconds) at which the bubble shape changes to the cusp shape can be estimated to be near the gel point. The relative error between the time and the gelation time is about $5.7 \%$. Meanwhile, for a large bubble volume $\left(100 \sim 800 \mathrm{~mm}^{3}\right)$, the time at which the shape transforms to cusp shape can be estimated to be at the point where the growth of the elastic property begins. The relative error between the time (e.g., 4800 seconds for $165 \mathrm{~mm}^{3}$ bubble) and the gelation time are bigger than that in the small bubble case. Because the forming mechanism of the cuspidal bubble with the long tail cannot be understood fully. Further study will be needed for the larger bubble cases.

\subsection{Classification of the bubbles by the shape regimes}

The bubble motion can be classified by the dimensionless numbers including Reynolds $(R e)$, Froude $(F r)$, and Weber ( $\mathrm{We}$ ). Then, we classified the observation of rising bubble into a diagram of shape regimes for bubbles in an unhindered gravitational motion through liquids with the use of dimensionless numbers, such as Re, Eötvös (Eo), and Morton $(M)$. Then a comparison was conducted between the experimentally observed shape of the bubble and the shape of the bubble was predicted from the classification of the bubbles by the shape regimes. The classification by $R e, E o$, and $M$, is essentially the same as the classification by $R e, F r$, and $W e$. This is because of the following relations: $E o=W e / F r$, $M=W e^{2} E o / R e^{4}$,

$$
R e=\frac{\rho_{L} u d_{b}}{\eta_{\mathrm{eff}}}, E o=\frac{\rho_{L} g d_{b}{ }^{2}}{\sigma}, \text { and } M=\frac{g \eta_{\mathrm{eff}}{ }^{4}}{\rho_{L} \sigma^{3}} .
$$

Here, $\eta_{\text {eff }}$ is the effective viscosity by applying effective shear

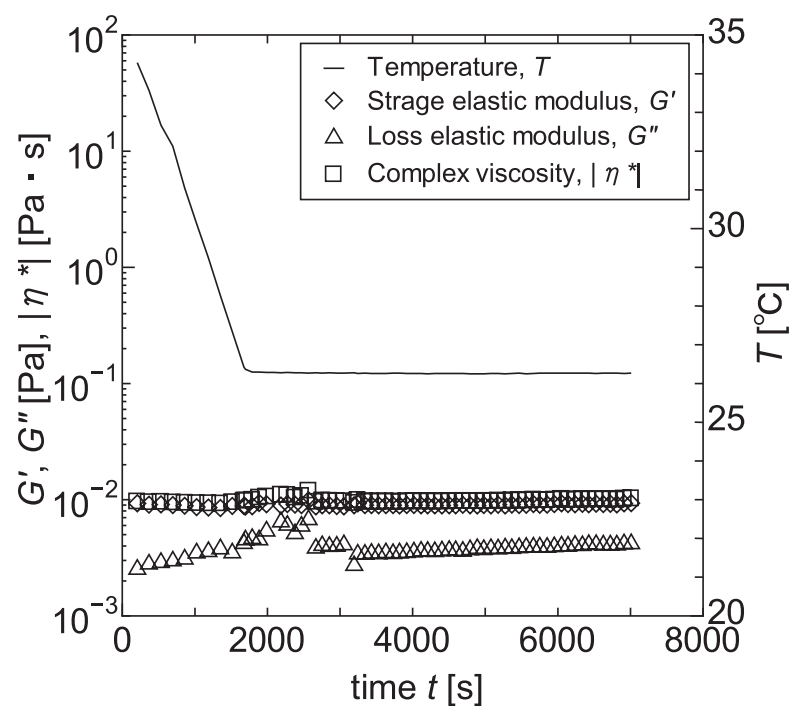

Fig. 7(a) Storage elastic modulus, $G^{\prime}$, loss elastic modulus, $G^{\prime \prime}$, and complex viscosity, $\left|\eta^{*}\right|$, for $3.0 \mathrm{wt} \%$ aqueous gelatin solution measured by the rheometer at $26^{\circ} \mathrm{C}$.

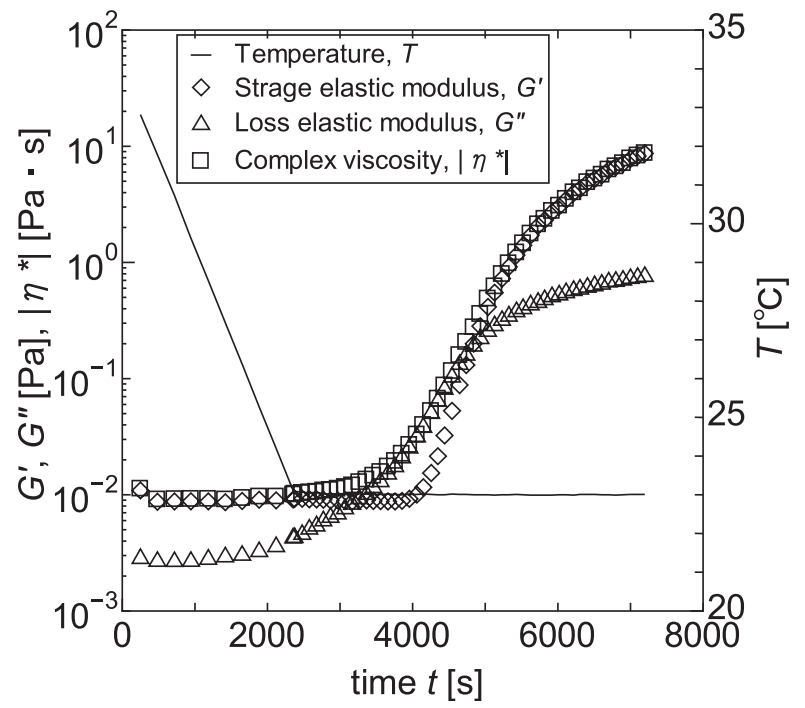

Fig. 7(b) Storage elastic modulus, $G^{\prime}$, loss elastic modulus, $G^{\prime \prime}$, complex viscosity, $\left|\eta^{*}\right|$, for $3.0 \mathrm{wt} \%$ aqueous gelatin solution measured by the rheometer at $23^{\circ} \mathrm{C}$

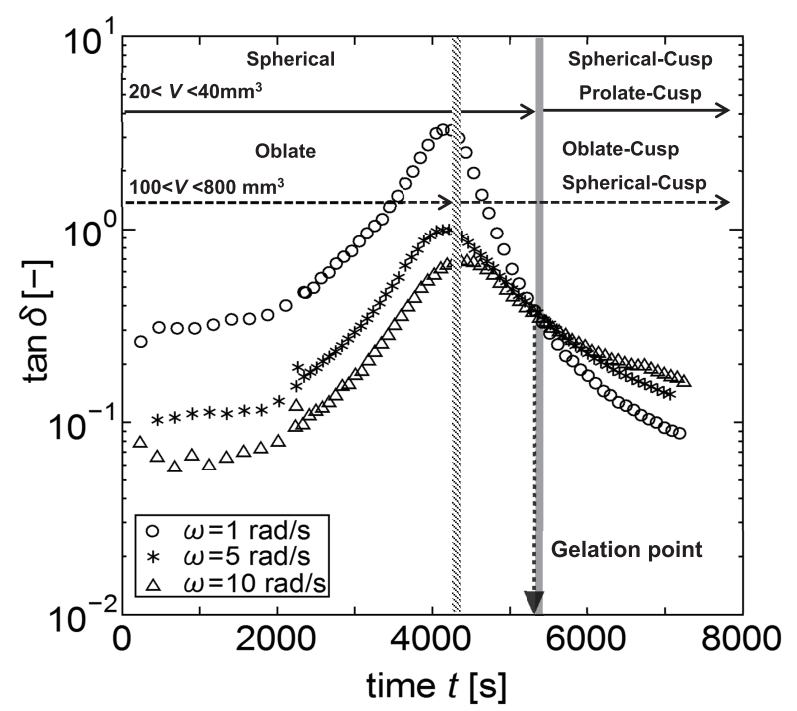

Fig. 8 Loss tangent versus gelation time of $3.0 \mathrm{wt} \%$ aqueous gelatin solution measured by the rheometer at $23{ }^{\circ} \mathrm{C}$. (Transition of bubble shapes superimposed) 
rate, $\dot{\gamma}_{\text {eff }}$ to Power-law model, $\eta=K(\dot{\gamma})^{n-1}$, where the effective shear rate, $\dot{\gamma}_{\text {eff }}$ is calculated by $\dot{\gamma}_{\text {eff }}=2 u / d_{b}{ }^{14)}$. The parameters of the power-law model are presented in Table V. It can be noticed that the value of the flow index is decreasing at long time zone. This may indicate that the large networks of macromolecules were built up, leading to the formation of the gel. This finding is corroborated with increasing value of the consistency factor. Associated with this observation, the Fig. 9 shows the variation of shear viscosity against the increasing time for the aqueous solution of gelatin. At initial period until 3500 seconds, the rheological properties can be considered to be close to the Newtonian fluid since there is no significant change in viscosity. Thereafter, the rheological properties are getting closer to the Non-Newtonian fluid due to the viscosity decrease with the increase of angular rate. This viscoelastic condition exhibits the same trend at 4000 seconds and at later time elapsed.

We have calculated the dimensionless numbers, $R e, E o$, and $M$, at each time for Runs 2, 3 and 4. For Run 2, the plot of the dimensionless numbers ( $R e$ versus $E o$ ) is shown in

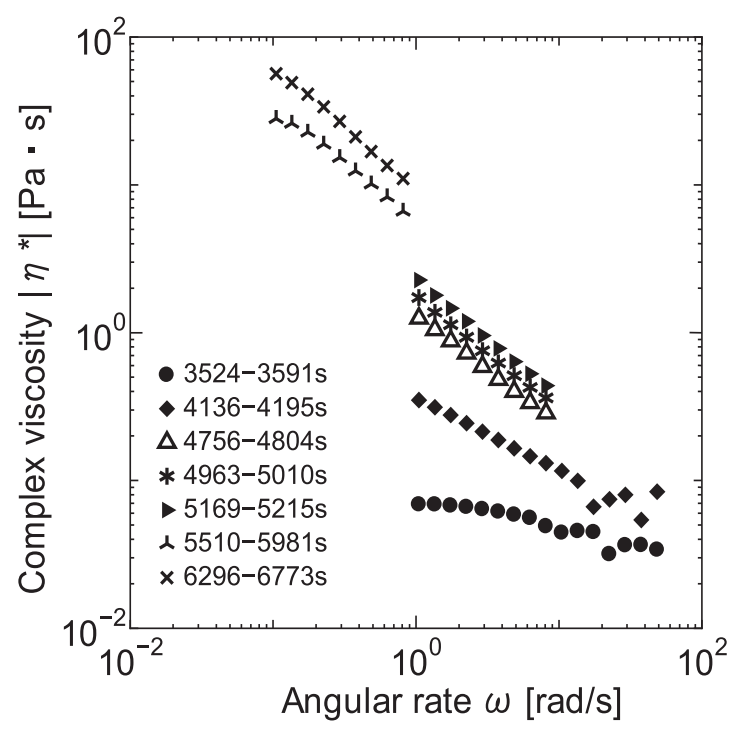

Fig. 9 Time change of rheological property of gelatin solution.
Fig. 10. There are five regimes of bubble shapes that can be identified. In regime I, the shape of the bubble is relatively spherical with slight deformation that happens at any values of $E o$ against high values of $R e$. At high values of Re against high value of $E o$, the shape of the bubble is either a spherical cap with closed and steady wake or open and unsteady wake. At considerably high values of $R e$, the shape of the bubble becomes an oblate and wobbling or disk-like. The regime II covers the spherical shape of the bubble. When the spherical shape of the bubble is deformed with a cusp, it indicates the onset of Regime III. The bubble is skirted with a smooth and steady wake. In addition, the shape of the bubble can be skirted with wavy and unsteady skirt ${ }^{15)}$. The regime IV only encompasses the oblate cap shape of the bubble. Lastly, the regime $\mathrm{V}$ covers the oblate shape of the bubble that is wobbling.

The dimensionless numbers ( $R e$ versus $E o$ ) for Runs 3 and 4 were also computed and plotted in Fig. 11. Based on the physical observation of the rising bubble, the shape of the bubble is either spherical or oblate with the cusp depending on its size as represented in regime I. It is further noticeable that, from regimes II to IV, the shape of the bubble is oblate with the cusp, regardless of its size. Therefore, the use of a small bubble in the regime I is preferred to catch the gelation point.

\section{CONCLUSION}

This study successfully compared the shapes of a rising bubble with the viscoelasticity of the same aqueous gelatin solution characterized by a stress-controlled rheometer. The newly designed system using the rising bubbles presented a moderate disturbance during the physical gelling of gelatin solution under a conveniently temperature-controlled cooling system. It was found out that the change in bubble shape corresponds to the elastic behavior of solution due to the

Table V Time change of model parameters of Power-law model.

\begin{tabular}{|c|c|c|c|c|}
\hline $\begin{array}{c}\text { Time zone } \\
T[\mathrm{~s}]\end{array}$ & $\begin{array}{c}\text { Range of } \\
\text { angular rate } \\
\omega[\mathrm{rad} / \mathrm{s}]\end{array}$ & $\begin{array}{c}\text { Frequency } \\
f\left[\mathrm{~s}^{-1}\right]\end{array}$ & $\begin{array}{c}\text { Consistency } \\
K\left[\mathrm{~Pa} \cdot \mathrm{s}^{\mathrm{n}}\right]\end{array}$ & $\begin{array}{c}\text { Flow index } \\
{[-]}\end{array}$ \\
\hline $3524-3591$ & $1-50$ & $0.159-7.96$ & 0.00518 & 0.786 \\
\hline $4136-4195$ & $1-50$ & $0.159-7.96$ & 0.1476 & 0.534 \\
\hline $4756-4804$ & $1-10$ & $0.159-1.59$ & 0.3373 & 0.264 \\
\hline $4963-5010$ & $1-10$ & $0.159-1.59$ & 0.4267 & 0.235 \\
\hline $5169-5215$ & $1-10$ & $0.159-1.59$ & 0.5251 & 0.197 \\
\hline $5510-5981$ & $0.1-1$ & $0.0159-0.159$ & 1.5535 & 0.267 \\
\hline $6296-6773$ & $0.1-1$ & $0.0159-0.159$ & 2.0656 & 0.175 \\
\hline
\end{tabular}




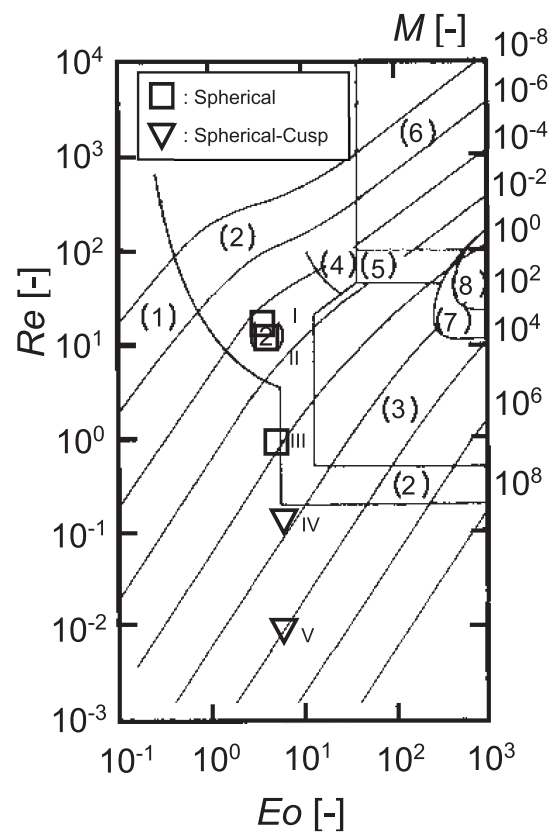

Fig. 10 Classification of the bubbles by the shape regimes for Run 2 ( $\square$ : Spherical, $\nabla$ : Spherical-Cusp, (1): Spherical (2): Oblate, Wobbling (3):Oblate-cap (4): Oblate, disk-like and wobbling (5):Sphericalcap with closed, steady wake (6): Spherical-cap with open, unsteady wake (7): Skirted with smooth, steady wake (8): Skirted with wavy, unsteady skirt) replotted from D.Bhaga and M.E.Weber ${ }^{15)}$.

progress in gelation process. The gelation point can be determined by detecting a change in the rising bubble shape from a sphere to prolate and cuspidal shape. It coincided with the time change in the bubble shapes with the tangent delta measurements. Lastly, it is recommended for measurement purposes that a small bubble is to be employed because of its less impact on the gelation structure around the bubble.

\section{ACKNOWLEDGEMENT}

The authors would like to thank Ms. Akiko Onishi and Mr. Keita Yamamoto for technical assistance with the experiments. This work was supported in part by JSPS KAKENHI Grant Numbers JP17K06152 and JP26420107.

\section{NOMENCLATURE}

d bubble diameter, $\mathrm{mm}$

$D$ depth of test section, $\mathrm{mm}$

E bubble aspect ratio

Eo Eötvös number

$f$ frequency, $\mathrm{Hz}$

$g$ gravity acceleration, $\mathrm{m} / \mathrm{s}^{2}$

$G$ relaxation elastic modulus, $\mathrm{Pa}$

$G^{\prime} \quad$ storage elastic modulus, $\mathrm{Pa}$

$G^{\prime \prime} \quad$ loss elastic modulus, $\mathrm{Pa}$

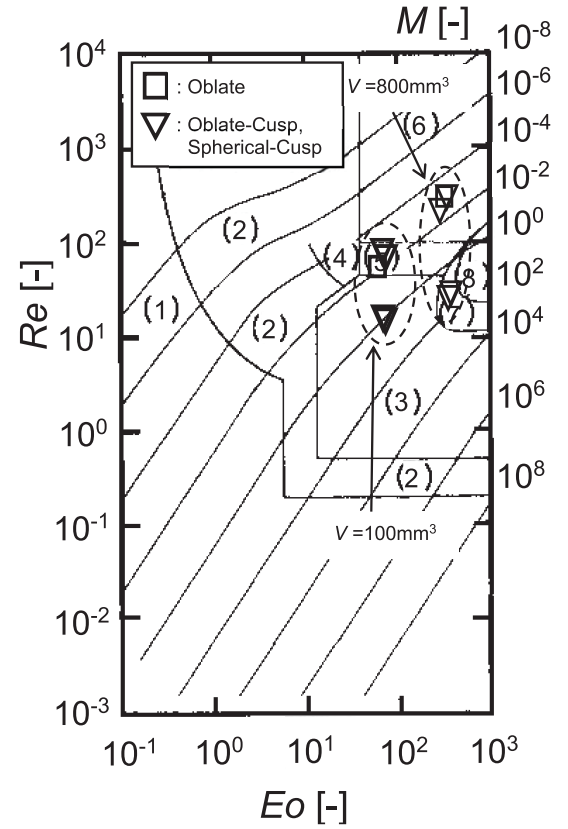

Fig. 11 Classification of the bubbles by the shape regimes for Run 3, 4 ( $\square$ : Oblate, $\nabla$ : Oblate-Cusp or Spherical-Cusp, (1):Spherical (2):Oblate, Wobbling (3): Oblate-cap (4): Oblate, disk-like and wobbling (5): Spherical-cap with closed, steady wake (6): Spherical-cap with open, unsteady wake (7): Skirted with smooth, steady wake (8): Skirted with wavy, unsteady skirt) replotted from D.Bhaga and M.E.Weber ${ }^{15}$.

$H \quad$ height of test section, $\mathrm{mm}$

$K$ model parameter in power-law model, $\mathrm{Pa} \cdot \mathrm{s}^{\mathrm{n}}$

$M \quad$ Morton number

$n \quad$ model parameter in power-law model

Re Reynolds number

$T$ temperature, ${ }^{\circ} \mathrm{C}$

$t$ time, s

$u \quad$ rising velocity, $\mathrm{mm} / \mathrm{s}$

$V \quad$ volume, $\mathrm{mm}^{3}$

$W \quad$ width of test section, $\mathrm{mm}$

$\eta \quad$ viscosity, $\mathrm{Pa} \cdot \mathrm{s}$

$\left|\eta^{*}\right| \quad$ complex viscosity, $\mathrm{Pa} \cdot \mathrm{s}$

$\eta_{\text {eff }} \quad$ effective shear viscosity, $\mathrm{Pa} \cdot \mathrm{s}$

$\rho$ density, $\mathrm{kg} / \mathrm{m}^{3}$

$\omega \quad$ angular rate, $\mathrm{rad} / \mathrm{s}$

\section{REFERENCES}

1) Djaborov M, Leblond J, Papon P, J Phys France, 49, 319 (1988).

2) Zhou C, Yue P, Feng JJ, Phys Fluids, 18, 092105 (2006).

3) Homma S, Moriguchi K, Kim T, Koga J, J Chem Eng Japan, 47, 195 (2014).

4) Iwata S, Kato T, Menchavez RL, Fuji M, Mori H, Yoshitake Y, Yamada Y, Saiki Y, Ceram Int, 39, 5309 (2013).

5) Liu YL, Liao TY, Joseph DD, J Fluid Mech, 304, 321 (1995).

6) Liao Y, Lucas D, Chem Eng Sci, 65, 2851 (2010). 
7) Crabtree JR, Bridgwater J, Chem Eng Sci, 26, 839 (1971).

8) Guenet JM, "Thermoreversible Gelation of Polymers and Biopolymers", (1992), Academic Press, NY.

9) Shirota M, Sanada T, Arata Y, Watanabe M, Kameda M, Trans JSME, 73, 467 (2007).

10) Takahashi M, J Soc Rubber Industry, Japan 66, 237(1993).

11) Chiou B, Raghavan SR, Khan SA, Macromolecules, 34, 4526 (2001).
12) Jiao Y, Gyawali D, Stark JM, Akcora P, Nair P, Tran RT, Yang J, Soft Matter, 8, 1499 (2012).

13) Ohta M, Kobayashi N, Shigekane Y, Yoshida Y, Iwata S, J Rheol, 59, 303 (2015).

14) Ohta M, Iwasaki E, Obata E, Yoshida Y, J Non-Newtonian Fluid Mech, 116, 95 (2003).

15) Bhaga D, Weber ME, J Fluid Mech, 105, 61 (1981). 